\title{
Global Perspectives and Leadership
}

\author{
Oluremi B. Ayoko ${ }^{1,2}$ \\ ${ }^{1}$ Associate Professor of Management, UQ Business School, The University of Queensland and ${ }^{2}$ Editor in Chief, JMO
}

In Issue 26.4 (2020) of JMO, we examine two broad but related organizational phenomena: global perspectives and leadership. Organizations do not only concern themselves with their internal employees and local markets, but they are confronted with decisions that often impact their global employees and how global markets may impact their performance and productivity. In most cases, organisational leaders and middle level managers have to make some difficult decisions and use differing strategies to gain productivity and increase performance. These are complex situations that include topics such as strategic planning, national culture, subnational differences, internationalization, global collaboration, and leadership. In this Issue, we explore a diversity of perspectives around the above-named topics.

We commence with strategic planning and a paper by Guo, Zhou, Zhang, Hu and Song, that poses a question "Does strategic planning help firms translate slack resources into better performance?". Using data from 183 U.S. firms, the paper explores the connection between organizational slack and firm performance with different levels of strategic planning. The results suggest that at low levels of strategic planning the slack-performance relationship is linear, while at high levels of strategic planning this relationship is inverse- $U$ shaped. Organisations has a lot to learn from the outcome of this paper.

Our next paper takes a dynamic capability approach to further investigate the issue of strategic planning. In the paper, "International strategies of emerging market multinationals: A dynamic capabilities perspective", Deng, Liu, Gallagher and Wu focus on the importance of dynamic capabilities in shaping the nature of international strategies of emerging market multinationals from mid-range economies. The authors outline three internationalization strategies available to emerging market multinationals in their international expansion namely: sequential international ambidexterity (from exploitation to exploration), exploration to exploitation and structural international ambidexterity (simultaneous exploration and exploitation). Success factors associated with each of the ambidextrous internationalization strategies are also discussed.

Entry modes are critical for success with international markets. Here, Li, Burmester and Zámborský examine the "Subnational differences and entry mode performance: Multinationals in east and west China" as they compare the influence of entry mode choice on subsidiary performance in two regions of a developing host country. The result indicates that wholly owned subsidiaries outperform joint ventures in the developed region, whereas joint ventures outperform wholly owned subsidiaries in the less developed region. Additionally, the smaller performance gap between wholly owned subsidiaries and joint ventures in the developed region indicates that the magnitude of influence of entry mode choices on performance varies across subnational regions. Firms must therefore be more discriminating in formulating entry strategies to regionally heterogeneous countries.

Another comparative paper looks at Gender, individualism-collectivism and individuals' propensity to trust: A comparative exploratory study. In this article, Zeffane explores the impact of gender and individualism/collectivism on individual's propensity to trust in a sample from the United Arab Emirates. Results reveal that females are generally more collectivist and less trusting than their male counterparts. Further analyses reveal that small business/operators are generally 
more trusting than the other cohorts and the propensity to trust is also found to be strongly associated with collectivist (rather than individualist) aspirations.

National culture plays a significant part in global issues. In our next paper, National culture, employee empowerment and advanced manufacturing technology utilisation: a study of Nigeria and New Zealand, Obi, Leggett and Harris compare two national cultures providing more insight on how national culture and employee empowerment may advance manufacturing technology (AMT) utilization. Especially, the paper examines the role of national culture on managerial attitudes towards employee empowerment during AMT adoption. Outcomes show that the observed differences in AMT (such as empowerment) are attributable to different national values such that during AMT adoption, NZ's liberal culture encourages managers to empower employees than the Nigeria's authoritarian culture. With manufacturer organisations seeking investment opportunities in Africa, the paper is timely.

Continuing the subtheme of national culture, Nadeem and De Luque in the next paper investigate emerging markets in another national culture-Pakistan. Their paper, "Developing an understanding of human resource (HR)complexities in Pakistan with a GLOBE cultural lens present primary data from the GLOBE study in Pakistan and compares them with secondary data from another 61 GLOBE societies. Results show that the Pakistani society exhibits high power distance and in-group collectivism but low assertiveness and gender egalitarianism. There is also a desire to create a more egalitarian society. However, the lack of assertiveness and high in-group loyalty appear to be treasured in this society. More comparative cross-national studies are needed to develop a deeper understanding of the impact of culture on human resource practices in Pakistan and around the globe.

Still on the issue of national culture, the paper "Effects of work passion on turnover intention for Chinese government employees: The dualistic model of passion perspective" by Gong, Zhang, $\mathrm{Ma}$, Liu and Zhao investigates the relationship between work passion, subjective well-being, career adaptability and turnover intention for Chinese government employees. Results of the series of questionnaires indicate that harmonious passion is negatively related to turnover intention and that obsessive passion is positively related to turnover intention. Additionally, the relationship between harmonious/obsessive passion and turnover is mediated by subjective well-being, while career adaptability moderates the relationship between harmonious/obsessive passion and subjective well-being. JMO has a history of interest in studies that investigate how emotions impact employees' attitude and behavior. In this regard, another JMO paper on Happiness at work: Developing a shorter measure by Salas-Vanilla and Alegre is also a must read.

Our next paper takes a different tenor on global perspectives. This time around, it is about that collaboration. In their paper, "Global collaboration in knowledge intensive firms: The role of activity configurations", Hydle and Brock discuss how transnational knowledge intensive firms manage complexity across multiple locations, integrating various functional specializations and catering to multifaceted customer demands. Drawing on practice theory, the paper explores collaboration among experts across national borders and six configurations emerged: bilateral, trilateral, chain, star, network and co-location. These configurations separately relate to three interdependent axes: coordinated actions, interaction modes and spatial dimensions. The configurations expose the relevant integrative and responsive settings. The paper extends transnationality and practice theory by unpacking the complexity of transnational collaboration and service provision. Additionally, this paper extends the work of Anzola-Román and her team in Profiting from collaborative innovative practices: Identifying organisational success factors along the process also published by JMO.

From global perspectives to leadership, our second dominant theme in this issue is leadership. In the paper Do Leader Expectations Shape Employee Service Performance? Enhancing Self-Expectations and Internalization in Employee Role Identity, Binyamin investigates in two studies and using the Kelman's Theory of Social Influence as an anchor, how leaders influence employees' expectations and corresponding behaviors through three processes of social influence: 
compliance, identification, and internalization. Outcomes of the studies show that the leaders' normative expectations for high quality service enhance employee service performance not only by adjusting self-expectations to comply with an authority figure's expectations or by identification with the leader as a role model, but by a deep-rooted process where the leader's normative expectations are internalized into employee's role identity. Altogether, the paper extends both the theory of social influence and the theory of planned behavior.

In the next paper, Leveraging Leader-Leader Exchange to Enrich the Effect of Leader-Member Exchange on Team Innovation, Yang proposes that supervisors' upward leader-leader exchange relationships are an important boundary condition for the relationship between leader-member exchange (LMX) and a climate for innovation support at the group level. Specifically, the paper argues that the effect of resource spillover to poor-LMX subordinates within a work group is an important mechanism in the development of a climate that supports innovation. The findings indicate that the indirect effect of team LMX relationships on team effectiveness via a climate for innovation support is more positive under high levels of leader-leader exchange (LLX) but the effect is less positive under low levels of LLX.

Olsen and Solstad drill down to leadership at the middle organisational level. Their paper on Changes in the power balance of institutional logics: Middle managers' responses explores qualitatively how middle managers respond when an existing institutional logic is reinforced through radical organisational change. Contrary to findings in previous research, their data suggest that the middle managers choose to acknowledge available information about the managerial logic, and that they accept or reject the new power balance between the logics. Managers accept new power balance through loyalty or resignation and they reject by strategically adhering to the managerial logic as if they were a novice even when they should already be familiar with this logic.

Our next paper, The contribution of behavioral economics to crisis management decisionmaking by Parnell and Crandall argues that although scholarly work in the field of crisis management has flourished in recent years, the substantial and prospective applications from behavioral economics (e.g. from Herbert Simon to modern theorists) have yet to be systematically integrated into the literature. This paper presents a framework that categorizes applications from behavioral economics along three stages of the crisis management life cycle: crisis preparation, crisis action and post-crisis. It provides insights for scholars and practitioners into the crisis decision-making process and outlines why "less-than-rational" decision-making approaches often appear in crisis environments. This paper should be a staple read for managers who will be managing the COVID-19 crisis in their organizations.

Continuing on the topic of leadership, museums provide the context of our next paper. Museums are organizations that need to maintain relationships with several stakeholders in order to achieve their economic and social objectives. In our next paper, The mixed effects of organisational social capital and bridging ties: the case of museums, Camarero, Garrido and Hernández examine the effect of an organization's bonding social capital and a manager's social capital on the organization's ability to build external relationships. Results from the study indicate that the structure of internal social capital (cohesion and diversity) and the manager's role as a bridging tie facilitate relations with stakeholders and other museum networks. Moreover, collective social capital (bonding and bridging) has a direct impact on innovative proposals, museum's image and on fundraising.

Finally, the current issue ends on a methodological note. Mittal, Sengupta, Agrawal and Gupta in their paper "How prosocial is proactive: Developing and validating a scale and process model of knowledge-based proactive helping" are concerned with developing and validating a scale on knowledge-based proactive helping. Measuring knowledge-based proactive helping appears to be a missing link in this area of literature. Validated across multiple population, the scale reveals two factors: professional-development and problem-mitigation that represent the construct of proactive-helping. Also, a process model based on social exchange theory, social motivation 
theory and highlighting the psychosocial causes and benefits of proactive-helping is proposed. The study brings the pro-social and proactive exchanges to the forefront of knowledge-exchanges.

The fourteen articles we put together in our $4^{\text {th }}$ Issue of JMO for 2020 have covered a range of topics that provide significant insights into global issues and leadership in organizations. Altogether, these studies have shown that strategic planning, national culture/differences, crisis management, global collaboration, and leadership are crucial for organisations attempting to improve their leadership effectiveness and those attempting to go global with their products and services.

Cite this article: Ayoko OB (2020). Global Perspectives and Leadership. Journal of Management \& Organization 26, 391-394. https://doi.org/10.1017/jmo.2020.13 